\title{
Mass Spectrometry and Non-covalent Protein- Ligand Complexes: Confirmation of Binding Sites and Changes in Tertiary Structure
}

\author{
Sharon J. Shields and Olayinka Oyeyemi* \\ Chemistry and Materials Science, Lawrence Livermore National Laboratory, Livermore, California, USA
}

\section{Felice C. Lightstone and Rod Balhorn}

Biology and Biotechnology Research Program, Lawrence Livermore National Laboratory, Livermore, California, USA

An experimental approach is described for determining protein-small molecule non-covalent ligand binding sites and protein conformational changes induced by ligand binding. The methodology utilizes time resolved limited proteolysis and the high throughput analysis capability of MALDI TOF MS to determine the binding site in a tetanus toxin C-fragment (51 $\mathrm{kDa}$ )-doxorubicin (543 Da) non-covalent complex. Comparing relative ion abundances of peptides released from the time resolved limited proteolysis of tetanus toxin C-fragment (TetC) and the TetC-doxorubicin complex every $10 \mathrm{~min}$ from 10 to $120 \mathrm{~min}$ of digestion revealed that the binding of doxorubicin induced a significant change in surface topology of TetC. Four of the twenty-nine peptides observed by MALDI MS, including amino acids 351-360, 299-304, 305-311 and 312-316, had a lower abundance in the TetC-doxorubicin complex relative to TetC from 10 to $100 \mathrm{~min}$ of digestion. A decrease in ion abundance suggests doxorubicin obstructs the access of the protease to one or both termini of these peptides, identifying doxorubicin binding site(s). Conversely, five peptide ions, including amino acids $335-350,364-375,364-376,281-298$, and 316-328, all had a greater abundance in the digest of the complex, indicating an increase in accessibility to these sites. These five peptides flank regions of decreased ion abundance, suggesting that doxorubicin not only binds to the surface, but also induces a conformational change in TetC. (J Am Soc Mass Spectrom 2003, 14, 460-470) (c) 2003 American Society for Mass Spectrometry

$\mathrm{N}$ on-covalent complexes formed between biomolecules such as proteins, small molecules, DNA, RNA, and metal ions play a central role in many important biochemical processes such as gene transcription, cell signaling, and ion transport. Understanding molecular dynamics and the structural aspects of non-covalent interactions is also at the heart of drug design where therapeutic molecules work by blocking or modifying the biological function of proteins, DNA or RNA. Experimental design to probe biologically relevant non-covalent complexes must consider several aspects of complex formation including methods for (1) screening and identifying components of a complex, (2) mapping the interaction interface, and (3) measuring

Published online April 10, 2003

Address reprint requests to Dr. S. J. Shields, Chemistry and Materials Science, Lawrence Livermore National Laboratory, P.O. Box 808, L-231, Livermore, CA 94551 E-mail: shields9@llnl.gov

*Current address: Department of Chemistry, University of California at Berkeley, Berkeley, California, USA. consequences of complex formation on secondary, tertiary, or quaternary structure.

During the last decade, electrospray ionization (ESI) and matrix assisted laser desorption ionization (MALDI) mass spectrometry (MS) have become viable techniques for measuring the three aforementioned aspects of non-covalently bound macromolecular complexes. ESI, in particular, has emerged as the ionization method of choice for detection of intact non-covalent complexes [1-5] because it involves molecular ionization directly from aqueous-based solutions designed to mimic physiological conditions. ESI gently evaporates the solvent, leaving solution based macromolecular complexes intact while transforming the complexes into gas-phase ions. For example, Wigger et al. [5] combined ESI FT-ICR MS with stored-waveform inverse Fourier transform and IR multiphoton dissociation to detect intact protein-ligand complexes from a solution containing a protein and a combinatorial library of 324 peptides. Following detection of the ligand free protein and intact non-covalent complexes, selective dissocia- 
tion via gas-phase methods identified the ligand(s) bound to the protein.

Ancillary techniques involving immobilized proteins [6], pulsed ultrafiltration [7], affinity chromatography $[8,9]$ or size exclusion chromatography $[10,11]$ as a means for isolating members of molecular complexes prior to MS analysis have also been developed to screen for potential protein-ligand complexes. Unlike the previous examples, these techniques do not rely on ionization and detection of intact complexes. For example, Cancilla and co-workers [6] developed an immobilized enzyme MS method to screen for potential small molecule inhibitors of pepsin and glutathione S-transferase from a combinatorial library consisting of 17-20 ligands. In this study, MS was performed on a chosen molecular library prior to and following incubation with immobilized enzymes. Differences in relative ion abundances of the library molecules prior to and after incubation not only identified inhibitors that bound to the enzyme, but also reflected the affinity of the molecules for the protein. Size exclusion gel filtration chromatography offers another means of identifying protein-ligand complexes by allowing large proteins and protein-ligand complexes to pass through a column while retaining lower molecular weight compounds. ESI MS performed under denaturing conditions, of the filtrate identified ligands bound to the protein [10, 11]. The methods described above for screening and identifying protein-ligand complexes only determine whether a given ligand binds to a protein, but does little to indicate binding sites or protein structural changes induced by complex formation.

Recently, covalent protein crosslinking coupled to MS analysis has been used to obtain low resolution tertiary structural information of proteins via intramolecular crosslinking [12] and to determine binding sites in protein-ligand and protein-protein complexes via intermolecular crosslinking [13-17]. Shapiro et al. [17] defined the ganglioside binding region of tetanus toxin C-fragment by covalently crosslinking ganglioside GD1b modified with a radio-labeled photoaffinity tag on the terminal sialic acid. After covalently attaching the modified ganglioside to TetC, the complex was subjected to proteolysis and the resulting fragments separated on SDS-PAGE gels. In-gel digestion followed by MALDI MS of the band containing the radiolabeled photoaffinity tag identified the C-terminal 34 amino acids of the 452 amino acid protein as the GD1 binding site. Although covalent crosslinking may provide useful information to indicate binding sites by formation of covalent bonds, the true dynamic nature of protein structural changes induced by complex formation are difficult to probe. For example, the photoaffinity tag only identified the point at which the one labeled sialic acid interacts with protein and does not address possible interactions with the remainder of the ganglioside structure.

NMR and X-ray crystallography are the primary analytical tools used to obtain atomic level structural information relating to protein-ligand binding sites and protein conformation changes induced by complex formation. Recently, however, limited proteolysis coupled to MS has been shown to provide important structural information of solution-phase non-covalent interactions, albeit low resolution, by determining structural intermediates in protein folding studies [18, 19] and mapping interfaces in protein-protein [20-24] and protein-DNA complexes [25]. Limited proteolysis relies on the ability of a protein to interact with the structural motif surrounding a protease's active site [26], i.e., a protease cannot cleave a protein if a cleavable site is protected by a protein's tertiary structure or by complex formation with another molecule. For example, Cohen et al. [25] utilized limited proteolysis and MALDI MS to determine the interaction interface between the transcription factor Max and a Max-specific DNA sequence. Using peptide maps generated with MALDI MS, they observed a significant decrease in proteolysis rates and changes in proteolysis patterns in the Max-DNA complex relative to Max alone. The difference in peptide maps and digestion rates were used to infer the interfacial region of the complex and to suggest protein conformational changes. Limited proteolysis and MALDI MS analysis were also used by Kriwacki et al. [20] to ascertain the binding sites in a protein-protein complex. In this example, the authors interpreted the absence of peptide ions in the mass spectrum of the protein-protein complex relative to free protein to indicate the region of binding. However, based on limited proteolysis and MS experiments alone, it was difficult to unambiguously discern the binding interface of the protein-protein complex versus potential conformation changes induced by complex formation. To find binding sites and detect conformation changes, the above examples exploit significant changes in protein folding or high affinity complexes with large interfaces to completely block a protease's access to specific amino acids. To date, limited proteolysis and MS methods have not been used to understand structural impacts of protein-small molecule ligand non-covalent complex formation.

Experimental screening of small molecules that bind to target proteins while obtaining detailed structural information about binding sites and protein tertiary structure changes is critical to the development of therapeutic agents as well as development of chemical sensors based on molecular recognition principles. In this paper, we demonstrate the use of time resolved limited proteolysis of the non-covalently bound TetCdoxorubicin complex using MALDI TOF MS to determine doxorubicin binding site(s). MALDI TOF MS was chosen as the analysis technique over ESI or LC/MS because of the high sample throughput and automation necessary to collect statistically relevant data. In addition, we utilized ESI MS to demonstrate TetC-doxorubicin non-covalent complex formation at solution conditions optimized for limited proteolysis.

Tetanus neurotoxin (TeNT) is a $150 \mathrm{kDa}$ clostridial 
toxin consisting of $50 \mathrm{kDa}$ and $100 \mathrm{kDa}$ polypeptide chains linked by a disulfide bond. The heavy chain is known to bind to presynaptic neuronal cells followed by light chain penetration into the cell which inhibits neurotransmitter release. The $51 \mathrm{kDa}$ carboxy-terminal domain of the heavy chain, TetC, has been shown to be the binding subunit [27] to gangliosides on the neuronal cell surface [28]. The first step to develop inhibitors of TeNT function or to develop chemical sensors based on recognition principles is to find molecules that bind to the protein and inhibit binding to gangliosides on the cell surface. This requires an understanding of protein structure and protein-ligand binding sites. Doxorubicin has been computationally predicted and experimentally shown to bind TetC $[29,30]$. Herein, we implement time resolved limited proteolysis of the non-covalently bound TetC-doxorubicin complex, using MALDI TOF MS analysis, to determine doxorubicin binding site(s). In addition to binding site determination, these methods also suggest the ability to address protein dynamics by determining conformational changes induced by ligand binding.

\section{Experimental}

\section{Limited Proteolysis}

Time resolved limited proteolysis of TetC and TetCdoxorubicin were performed using sequencing grade modified trypsin (Promega, Madison, WI) in a 300:1 protein:enzyme wt:wt ratio. TetC $(4 \mu \mathrm{M})$ and a TetC: doxorubicin mixture $(4: 40 \mu \mathrm{M})$ in $10 \mathrm{mM}$ ammonium acetate ( $\mathrm{pH}$ 7.7) were digested simultaneously. A $1 \mu \mathrm{L}$ aliquot of each digest was extracted every $10 \mathrm{~min}$ up to $120 \mathrm{~min}$, followed by every $60 \mathrm{~min}$ up to $12 \mathrm{~h}$. To quench trypsin activity, each $1 \mu \mathrm{L}$ aliquot was added to $6 \mu \mathrm{L}$ of $0.1 \%$ TFA ( $\mathrm{pH} 1.9$ ).

\section{MALDI MS Analysis}

MALDI mass spectra were acquired on a PerSeptive Biosystems (Framingham, MA) Voyager DE-STR TOF mass spectrometer equipped with a nitrogen laser (337 $\mathrm{nm}$ ). Mass spectra were obtained in positive ion and reflectron mode with an acceleration voltage of $25 \mathrm{kV}$, grid voltage of $64.8 \%$, and delay time of $275 \mathrm{~ns}$ and averaged over 100 laser shots. Each spectrum was internally calibrated with TetC tryptic fragment ions at $\mathrm{m} / \mathrm{z} 2344.1131$ and 1135.5132 providing mass measurement accuracies of approximately $10 \mathrm{ppm}$ across the 600-5500 Da mass range.

For each time point in the time resolved limited proteolysis, ten total mass spectra were acquired, five from two different sample spots resulting in 440 total mass spectra, 220 from the $\mathrm{TetC}$ and 220 from the TetC-doxorubicin digests. Absolute ion abundances of the tryptic fragments generated by MALDI, as determined by peak area of the monoisotopic peak were, on average, $\pm 20 \%$ (see sample preparation below). Due to the inherent inconsistency in ionization and ion yield in MALDI, the data is represented in percent relative abundance. To calculate an average relative ion abundance and associated error with this measurement, a total ion current for each mass spectrum $(\mathrm{m} / z$ 600-5500) was determined by summing monoisotopic peak areas for each peptide ion observed. Peak areas of each ion were then divided by the total ion current, providing a percent relative ion abundance of each ion in each mass spectrum. The percent relative abundance was averaged for the 10 spectra collected at each time point in the limited proteolysis.

Ion yields vary significantly from shot to shot in MALDI; therefore, uniform and reproducible MALDI sample preparation techniques are critical to the success and reproducibility of the limited proteolysis experiments. The typical dried droplet method of co-depositing analyte and matrix from a $50 \%$ organic solution onto a metal surface causes inhomogeneous and irregular crystallization resulting in analyte segregation and erratic ion yields even within the same sample spot [31]. Several MALDI sample deposition methods have been developed to promote uniform analyte deposition on a metal surface [32-34]. We utilized a MALDI sample preparation system outlined by Edmondson and Russell [33]. Briefly, in our study, $5 \mu \mathrm{L}$ of a saturated solution of $\alpha$-cyano-4-hydroxycinnamic acid in methanol was deposited on a flat stainless steel MALDI sample plate. Fast evaporation of methanol deposited a uniform matrix crystal seed layer. $3 \mu \mathrm{L}$ of $5 \mathrm{mg} / \mathrm{mL}$ $\alpha$-cyano-4-hydroxycinnmaic acid in methanol was combined with the $1 \mu \mathrm{L}$ of tryptic digest and $6 \mu \mathrm{L}$ of $0.1 \%$ TFA and $0.5 \mu \mathrm{L}$ of this mixture was deposited on the matrix crystal bed. To assure that the ion yield and ion distribution is representative of the entire sample spot, the laser was rastored across the sample spot, averaging 100 laser shots per spectrum. Although the absolute ion abundances acquired from this homogeneous sample deposition was on average $\pm 20 \%$, the relative ion yield and ion distribution across the entire spot was consistent.

Ion yields in MALDI can also be influenced by the molecular composition of the sample. To verify that uncomplexed doxorubicin was not responsible for ion suppression/enhancement effects in determining relative ion abundances, a control experiment was performed. TetC was digested as described above and doxorubicin was added post digestion in a TetC:doxorubicin 1:10 molar ratio. Relative peptide ion abundances were calculated for the TetC digest and the TetC digest-doxorubicin mixture. At the early stages of proteolysis, 10 and $20 \mathrm{~min}$, doxorubicin had a suppression effect on the absolute abundances of all ions observed, but relative ion abundances in the sample containing doxorubicin were within the experimental error of those in the control sample. As more tryptic peptides were liberated from TetC at longer digestion times, doxorubicin had less impact on ion suppression. Ion enhancement due to the presence of doxorubicin was 
never observed. Therefore, the relative ion abundances determined in the limited proteolysis studies of TetC and TetC-doxorubicin reflects changes in digestion rates in solution, and not ion suppression/enhancement effects of MALDI.

\section{ESI MS}

ESI TOF MS (LCT, Micromass, Manchester, UK) methods were utilized to detect non-covalent complex formation between TetC and doxorubicin under sample conditions optimized for binding site determination by limited proteolysis. Similar ESI TOF MS experiments on TetC were performed by Lightstone et al. [29], but have been reproduced and presented here to address structural aspects of non-covalent complex formation as detected by ESI MS. Briefly, lyophilized TetC (Roche Molecular Biochemicals, Indianapolis, IN) was reconstituted in $10 \mathrm{mM}$ ammonium acetate at $\mathrm{pH} 7.7$ to a concentration of $38 \mu \mathrm{M}$ and doxorubicin (Sigma-Aldrich Chemical Co.; St. Louis, MO) to a concentration of $0.9 \mathrm{mM}$. A final mixture containing $10 \mu \mathrm{M}$ TetC and 100 $\mu \mathrm{M}$ doxorubicin was allowed to equilibrate at room temperature for $30 \mathrm{~min}$ prior to ESI analysis. Instrument conditions consisted of $3000 \mathrm{~V}$ applied to the needle and a cone voltage that was varied from $80-120 \mathrm{~V}$ with an infusion rate of $3 \mu \mathrm{L} / \mathrm{min}$. Mass spectra were averaged over 50 scans. Optimum cone voltages for observing complex formation were 90-100 V and complete complex dissociation was observed at $120 \mathrm{~V}$.

\section{Results and Discussion}

Understanding where small molecules bind to proteins and how the binding affects the protein structure is the key to the development of high affinity ligands for the purpose of immunoassay detection technologies or drug discovery. NMR and X-ray crystallography have been the primary analytical tools used to obtain detailed atomic-level characterization of protein-ligand interactions. Computational modeling using homology [35], docking or solvation [36], or grid [37] based methods is also playing a key role in identifying regions on proteins that may serve as a binding site as well as predicting what molecules will bind to that site. In this study, we have developed a high throughput time resolved limited proteolysis MALDI MS method to confirm doxorubicin's computationally predicted binding site to TetC [29]. TetC-doxorubicin is a weakly bound non-covalent complex with an experimentally determined dissociation constant of $9.4 \mu \mathrm{M}$ [29]. Since doxorubicin (543 Da) is not likely, nor predicted, to induce a global conformation change in TetC $(51.7 \mathrm{kDa})$ and the interaction interface is small, meticulous experimental design and data interpretation make time resolved limited proteolysis unique to the study of weakly bound protein-small molecule non-covalent complexes.

Immobilized enzyme mass spectrometry techniques
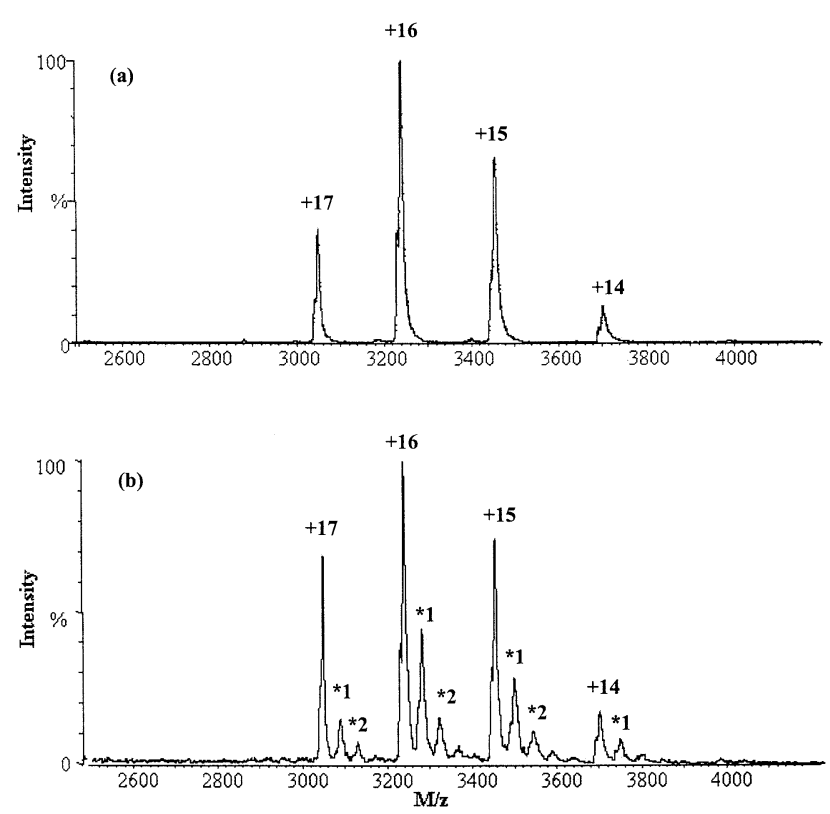

Figure 1. ESI-TOF mass spectrum of (a) TetC and (b) a solution containing a 1:10 molar ratio of TetC:doxorubicin. The peaks labeled ${ }^{*} 1$ and ${ }^{*} 2$ in (b) correspond to [TetC +1 doxorubicin + $\mathrm{nH}]^{\mathrm{n}+}$ and $[\text { TetC }+2 \text { doxorubicin }+\mathrm{nH}]^{\mathrm{n}+}$, respectively. Although binding of one, two, and perhaps three doxorubicin molecules to TetC is observed, the overall charge state distribution is conserved in the mass spectrum of the non-covalent complex.

for screening molecular libraries of potential small molecule inhibitors to enzymes [6] utilized excess enzyme relative to the total inhibitor molar concentration to be certain there are plenty of protein sites available for binding. The limited proteolysis experiments require the reverse scenario, molar excess ligand, to be certain that as many binding sites as possible are occupied (i.e., no excess ligand-free protein in solution) without forcing non-specific interactions to occur. Initially, molar ratios were varied from 1:1 to 1:50 TetC: doxorubicin. At a 1:1 molar ratio there was no quantitative difference in the proteolytic fragment ion abundances, as measured by MALDI MS, observed from the TetC relative to the TetC-doxorubicin complex. This was likely due to a large amount of unbound TetC in the TetC-doxorubicin solution. Conversely, at a 1:50 TetC:doxorubicin ratio there was a significant change in the proteolysis patterns suggesting that when present in great excess, doxorubicin interacted nonspecifically with the surface of TetC or generally interfered with the protease's access to TetC. Changes in the rate of proteolysis were first observed at a 1:10 TetC: doxorubicin molar ratio, and we chose this molar ratio to develop the time resolved limited proteolysis methods.

NMR [30] and ESI MS [29] ligand screening techniques have previously demonstrated that doxorubicin does bind to TetC, but could not determine binding sites. In particular, titration experiments utilizing ESI MS experiments were used to determine TetC-doxorubicin dissociation constants [29], but have been repro- 
1 MKNLDCWVDN EEDIDVILKK STILNLDINN DIISDISGFN SSVITYPDAQ

51 LVPGINGKAI HLVNNESSEV IVHKAMDIEY NDMENNETVS FWLRVPKVSA

101 SHLEQYGTNE YSIISSMKKH SLSIGSGWSV SLKGNNLIWT LKDSAGEVRQ

151 ITFRDLPDKF NAYLANKWVE ITITNDRLSS ANLYINGVLM GSAEITGLGA

201 IREDNNITLK LDRCNNNNQY VSIDKERIFC KALNPKEIEK LYTSYLSITF

251 LRDFWGNPLR YDTEYYLIPV ASSSKDVQLK NITDYMYLTN APSYTNGKLN

301 IYYRRLYNGL KEIIKRYTPN NEIDSFVKSG DEIKLYVSYN NNEHIVGYPK

351 DGNAFNNLDR ILRVGYNAPG IPLYKKMEAV KLRDLKTYSV QLKLYDDKNA

401 SLGLVGTHNG OIGNDPNRDI LIASNWYFNH LKDKILGCDW YFVPTDEGWT

451 ND

Figure 2. 452 amino acid sequence of TetC. The underlined amino acids are observed in the MALDI TOF mass spectrum of the tryptic digest of TetC and the TetC-doxorubicin complex by 120 min.

duced here to simply demonstrate ligand binding at the same solution conditions optimized for limited proteolysis experiments. Figure 1 contains the ESI TOF mass spectrum of TetC (Figure 1a) and TetC-doxorubicin complex(es) in a 1:10 molar ratio (Figure 1b). In the ESI mass spectrum revealing TetC-doxorubicin complex formation, adducts to TetC correspond to the binding of one (peaks labeled with *1) and two (peaks labeled with *2) doxorubicin molecules. If the assumption is made that gas-phase ESI-MS mimics solution-phase behavior, then this binding stoichiometry, at a 1:10 TetC:doxorubicin molar ratio, suggests multiple doxorubicin binding sites and/or non-specific binding. However, it is difficult to quantitate the binding stoichiometry by ESI MS [38] in this example because protein denaturization due to solvent evaporation in the electrospray process in combination with excess ligand can affect proteinligand binding stabilities, binding sites and binding stoichiometry. Others have noted the inability to directly correlate energetics of gas-phase to solutionphase binding in protein-ligand complexes. $\mathrm{Wu}$ et al. [4], Wigger et al. [5], and Rogniaux [43] et al. observed that gas-phase stabilities of protein-ligand complexes reflect polar and electrostatic interactions in contrast to solution based stabilities which are dominated by hydrophobic interactions, signifying the importance of understanding solvation effects in non-covalent complex formation when designing MS experiments. Moreover, the ESI experiments presented here were not intended to determine ligand binding specificity or non-specificity, but simply to demonstrate binding. To determine ligand binding specificity by ESI MS methods several experimental conditions would need optimization including cone voltages, ESI source temperatures, flow rates (e.g.; nanospray), solution ionic strength, $\mathrm{pH}$, and ligand and protein titration experiments.
Solution based NMR experiments used to detect doxorubicin binding to TetC, a 1:1 protein:ligand molar ratio was not sufficient to detect ligand binding and optimal molar ratios were 1:16 to 1:22 [30]. In ligand competition experiments using NMR, another ligand predicted to bind to the same site as doxorubicin, 3'-sialyllactose, displaced doxorubicin from TetC suggesting a specific interaction. However, the NMR experiment, as performed, could not rule out additional, low abundant non-specific doxorubicin binding. In a similar manner, the limited proteolysis studies are used to probe solution-phase binding and excess ligand was required to observe changes in the rates of protein digestion when coupled to MALDI MS analysis.

Independent of the energetics of gas-phase and solution-phase non-covalent complex formation, information about the conformation of $\mathrm{TetC}$ can also be ascertained from the charge state distributions in the ESI mass spectra of Figure 1. Protein charge state distributions in ESI have been shown to be dependent on solution conditions such as $\mathrm{pH}$ and organic content where changes in the charge state distribution reflects denatured versus globular protein structure [39-42]. In regards to protein-ligand non-covalent complexes, Pramanik et al. [2] observed a shift to a lower charge state distribution $(+13$ to +9$)$ of the ras protein-guanosine diphosphate (GDP) complex at a $\mathrm{pH}$ of 4.7 relative to a +10 to +8 charge state distribution of the complex at $\mathrm{pH}$ 5.2. At both $\mathrm{pHs}$, the ras-GDP complex remained intact even though the charge state distribution suggested a slight denaturation of the protein. Based on the concept that changes in charge state distributions may reflect protein conformational changes in solution, the absence of a shift in the charge state distribution of the TetC-doxorubicin complex relative to TetC, implies that complex formation did not have a significant impact on the global structure of TetC. Thus, the ESI MS data 


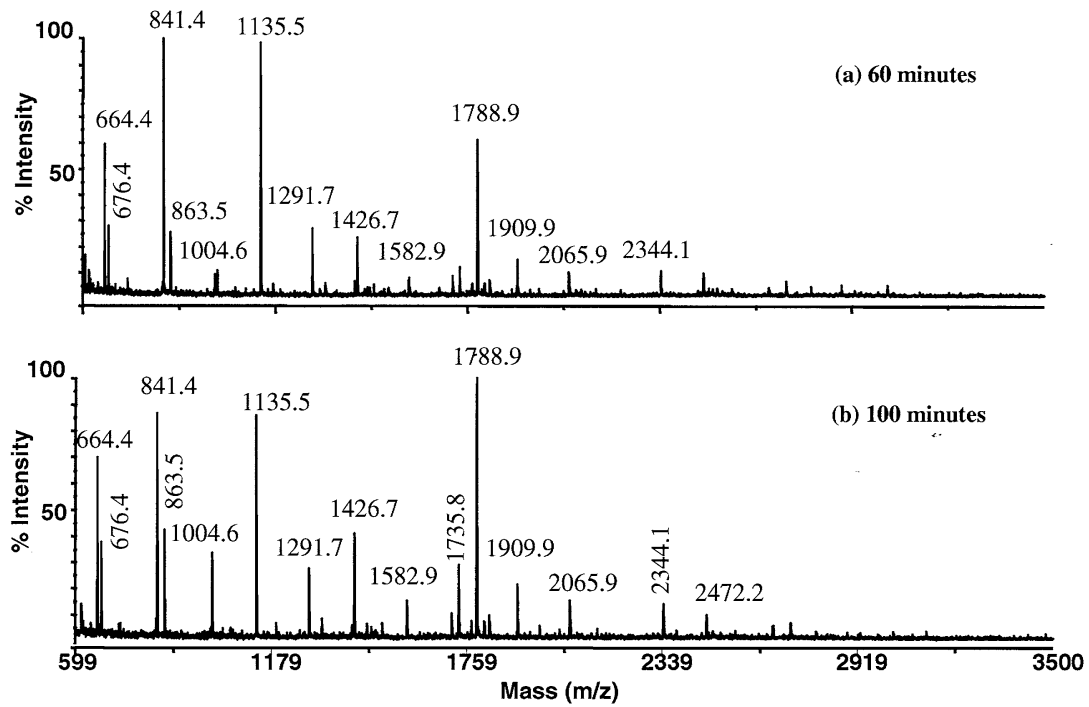

Figure 3. MALDI-TOF mass spectrum of a (a) 60 min tryptic digest and (b) 100 min tryptic digest of TetC.

suggests doxorubicin binds to TetC with an insignificant amount of tertiary structure change to affect the charge state distribution, but does not provide any information as to the binding site(s).

Since doxorubicin is predicted to bind to the surface of TetC and not induce a global conformation change, an understanding of the surface topology of TetC relative to the TetC-doxorubcin complex is imperative in order to determine binding site(s) and this is accomplished with time resolved limited proteolysis. Trypsin was selected as the protease because there are several lysine and arginine residues in the predicted doxorubicin binding region and a 300:1 TetC:trypsin wt:wt ratio provided a proteolysis rate that was measurable on the time scale of the experiment. Figure 2 contains the 452 amino acid sequence of TetC where the lines indicate all peptides observed within the first 120 min of digestion accounting for $57 \%$ sequence coverage. Proteolytic fragments overlapping in amino acid sequence from $304-$ 328 and 364-376 aid in the interpretation of specific amino acids affected by TetC-doxorubicin complex formation.

MALDI MS analysis of the time resolved limited proteolysis experiments (every $10 \mathrm{~min}$ for $120 \mathrm{~min}$ ) are represented in Figures 3 and 4 which show MALDI mass spectra of the peptide map generated by a trypsin digest at 60 and $100 \mathrm{~min}$ of TetC and the TetC-doxorubicin complex, respectively. These mass spectra are typical of the ten spectra obtained for each respective digest time. Quenching the tryptic digest with acid and
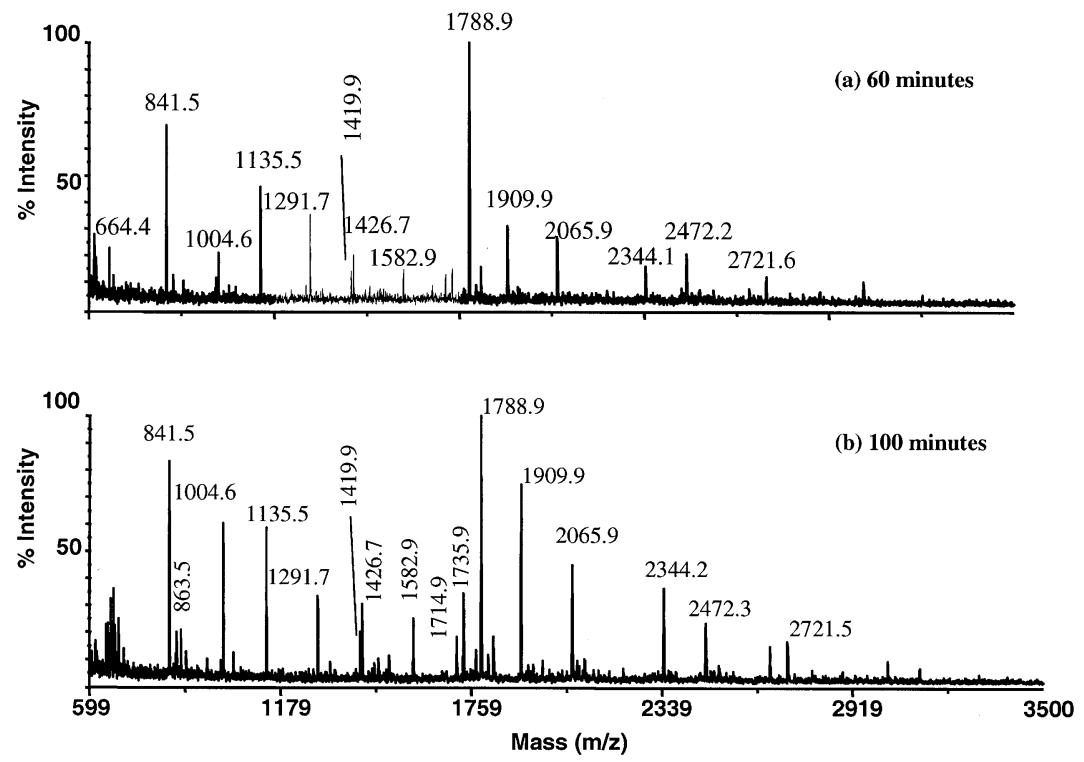

Figure 4. MALDI-TOF mass spectrum of a (a) 60 min tryptic digest and (b) 100 min tryptic digest of a solution containing 1:10 molar ratio of TetC:doxorubicin. 

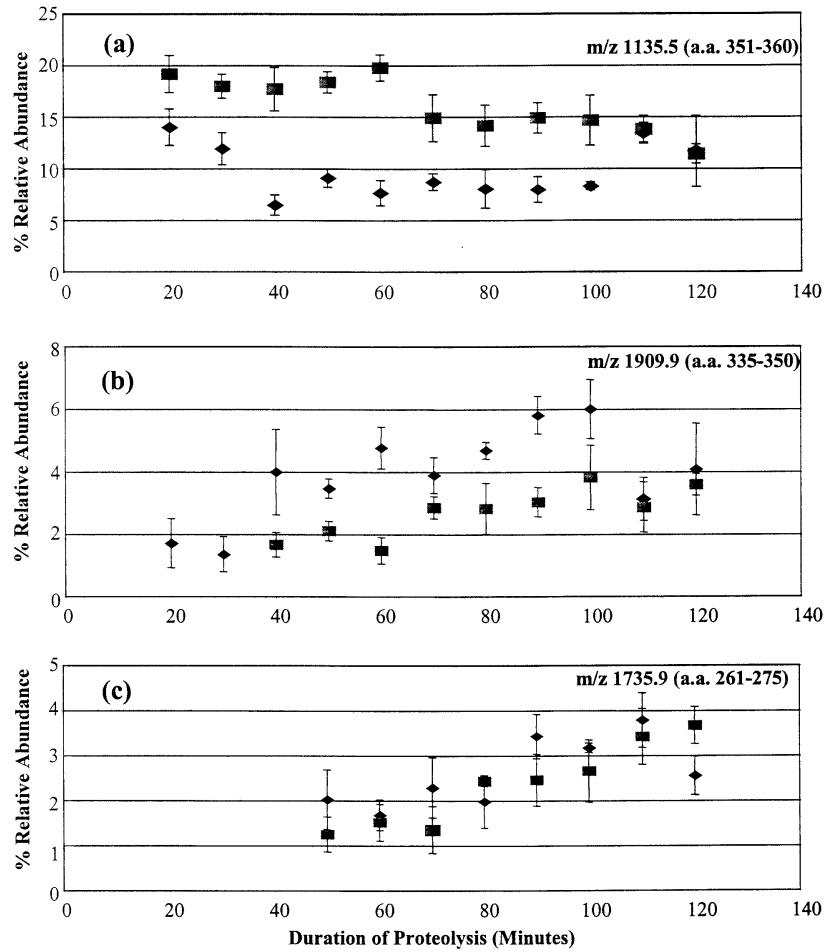

- TetC

- TetC-Doxorubicin Complex

Figure 5. Comparison of percent relative ion abundance versus digest time for various peptide ions observed in the TetC and the TetC-doxorubicin tryptic digests. (a) Peptide ion at $\mathrm{m} / \mathrm{z} 1135.5$ consistently has a decreased ion abundance in the TetC-doxorubicin complex from 20 to $100 \mathrm{~min}$. Three other peptides, $\mathrm{m} / \mathrm{z}$ 863.5, 676.4, and 841.5, follow a similar pattern. (b) Peptide ion at $\mathrm{m} / \mathrm{z}$ 1909.9 consistently has an increased ion abundance in the TetCdoxorubicin complex from 20 to $100 \mathrm{~min}$. Five additional peptides, $\mathrm{m} / \mathrm{z}$ 1291.7, 1419.9, 2065.9, 1520.9, and 1582.8, follow a similar pattern. (c) The ion abundance for peptide ion at $\mathrm{m} / \mathrm{z} 1735.9$ in the TetC-doxorbucin complex is within experimental error of the same ion in TetC. The same is true for 19 other peptides observed.

addition of MALDI matrix is likely to dissociate any specific non-covalent interactions remaining in solution after digestion; therefore, detection of doxorubicin bound to a tryptic peptide or TetC by MALDI MS is improbable. The peptide maps of $\mathrm{TetC}$ and the TetCdoxorubicin complex in the mass spectra of Figures 3 and 4 are qualitatively similar, which is expected because of the small interaction interface and lack of global protein conformation change in the TetC-doxorubicin complex. However, careful inspection of the MALDI mass spectra reveals significant differences in the rate of proteolysis at several amino acids. For example, in the proteolysis of the TetC-doxorubicin complex relative to TetC there is a reduction in the relative ion abundance of tryptic peptides at $m / z$ 1135.5, 863.5, 676.4, and 841.5. Figure 5a shows the statistical representation of the percent relative ion abundance versus proteolysis time for the peptide at $\mathrm{m} / \mathrm{z} 1135.5$ [amino acids (a.a.) D351-R360]. From 20 to $100 \mathrm{~min}$ this peptide is lower in abundance in TetC-doxorubicin
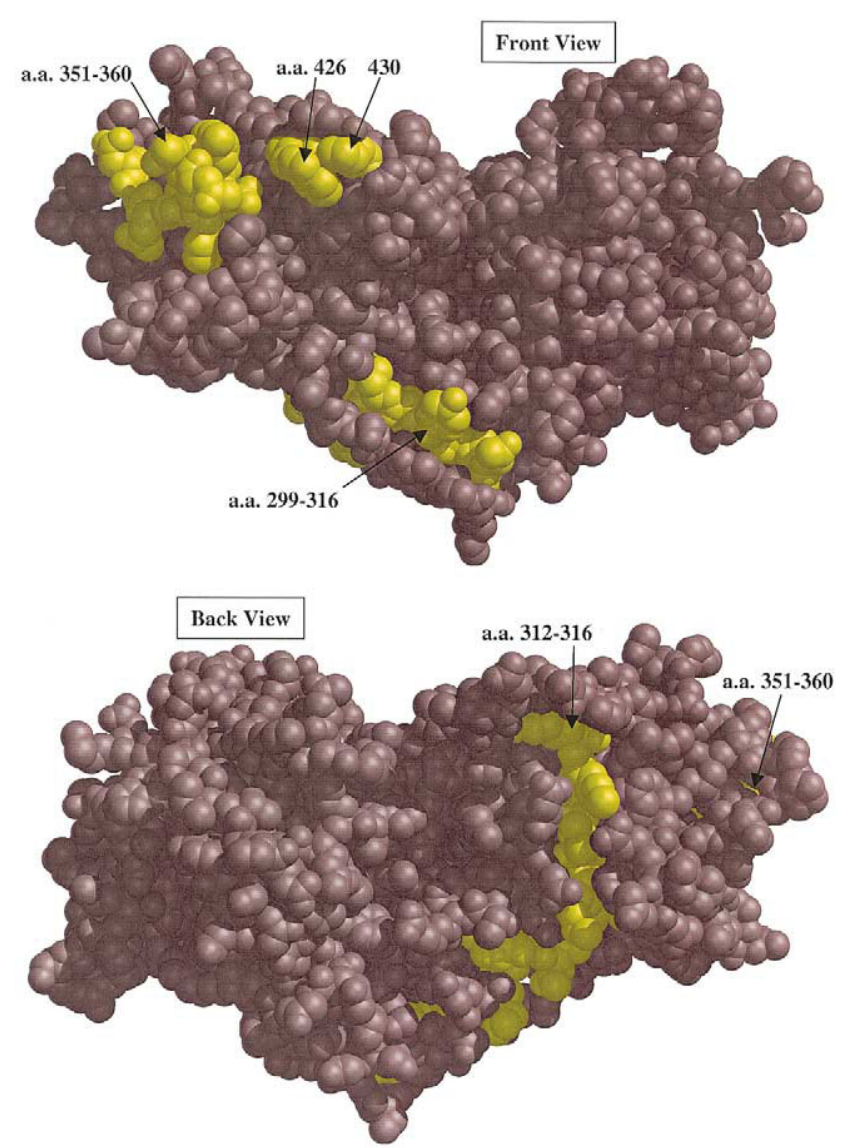

Figure 6. Space-filling molecular representation of TetC where the amino acids highlighted in yellow correspond to the tryptic peptides of lower abundance in the TetC-doxorubicin complex relative to TetC, including ions at $m / z 1135.5$ (a.a. 351-360), 863.5 (a.a. 305-311), 676.4 (a.a. 312-316), and 841.5 (a.a. 299-304).

complex; at 110 and $120 \mathrm{~min}$, the relative ion abundance is within experimental error in both proteolysis experiments. Similar rates of digestion are observed for $\mathrm{m} / \mathrm{z}$

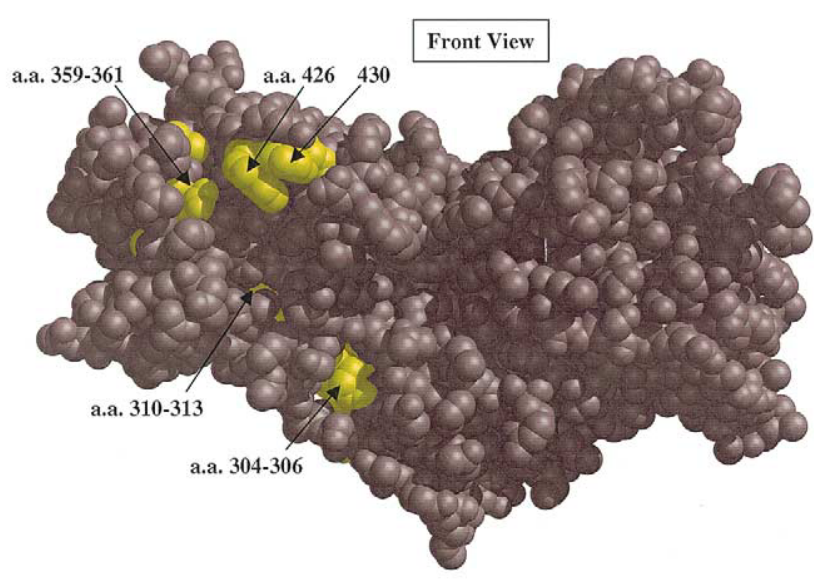

Figure 7. Space-filling molecular representation of TetC where the amino acids highlighted in yellow correspond to the individual amino acids of the peptides highlighted in Figure 6 that are responsible for the decrease of the peptide ion abundance. Doxorubicin is suggested to block trypsin from cleaving at amino acid R360, K311 and R305. 


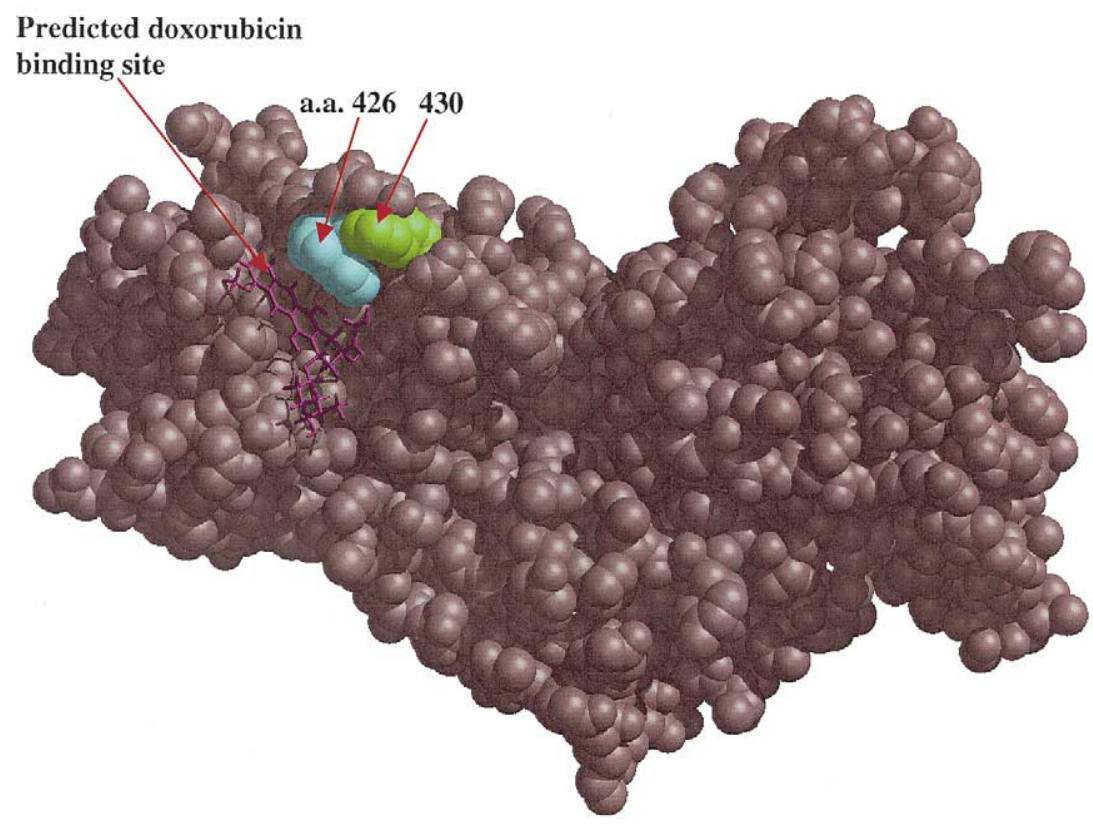

Figure 8. The computationally predicted doxorubicin binding site.

863.5 (a.a. R305-K311), m/z 676.4 (a.a. F312-R316), and $\mathrm{m} / \mathrm{z} 841.5$ (a.a. L299-R304). The fact that the rates of proteolysis of these four peptides is reduced in the complex for the first $100 \mathrm{~min}$, but rebounds to equal that of the ligand-free protein at $110 \mathrm{~min}$ suggests that understanding the initial rates of proteolysis at the most accessible sites is critical because at longer times of digestion, the protease can access sites buried into the tertiary structure. One interpretation for the observation of peptides having a decreased abundance in the complex is that doxorubicin reduces the ability of the protease to access specific amino acids, such as K298, R304, R305, K311, R316, K350, or R360, by physically blocking these sites. Another possible interpretation is that upon binding, doxorubicin induces a conformational change that alters the tertiary structure enough to reduce the rate of proteolysis at a given site.

Another observation in the time resolved limited proteolysis of the TetC-doxorubicin complex relative to the ligand-free protein is an increase in relative ion abundance of six peptides including $\mathrm{m} / \mathrm{z} 1520.9$ (a.a. R305-R316), 1582.8 (a.a. R316-K328), 2065.9 (a.a. N281K298), 1291.7 (a.a. V364-K375), 1419.7 (a.a.V364-K376), and 1909.9 (a.a. L335-K350). Interestingly, the peptide ion at at $m / z 1520.9$ is a peptide with a missed cleavage site at amino acid K311. Because peptides containing R305-K311 and F312-R316 are less abundant in the complex, but R305-R316 is more abundant implies that the binding of doxorubcin interferes with trypsin's ability to access amino acids K311 and F312. A spacefilling molecular representation of the X-ray crystal structure of TetC (Figure 6) aids in the visualization of the peptides of decreased abundance in the complex. The yellow highlights, with exception of amino acids 426 and 430 , correspond to the four peptides of reduced ion abundance in the TetC-doxorubicin complex. The yellow highlighted amino acids in Figure 7 correspond to the peptide's terminal amino acids that could be blocked by doxorubicin, including D369-I361, L310I313, and R304-L306. If we compare the computationally predicted doxorubicin binding site (Figure 8) with the peptides that show a decreased abundance, it is consistent that doxorubicin blocks trypsin's ability to access R360 and K311, identifying one binding site. The reduced ion abundance of peptide L299-R304 has two possible explanations. One, doxorubicin has an additional binding site. This would corroborate the ESI MS data in Figure 1 that suggests doxorubicin has multiple binding sites at a 1:10 molar ratio. (Note, no attempt was made to design ESI experiments for characterizing ligand binding specificity). Or two, the specific binding of doxorubicin to the site near amino acids D369-I361 and L310-I313 induces a conformational change that reduces trypsin's ability to cleave at amino acids K298 or R304.

Surprisingly, the other five peptides exhibiting an increased relative ion abundance in the complex flank the regions where a decrease in ion abundance is observed. Figure $5 \mathrm{~b}$ contains the statistical representation of the percent relative ion abundance for peptide ion at $m / z$ 1909.9. (It is important to note that out of the 29 peptides observed in 120 min of the trypsin digest, 19 of the peptides did not exhibit a change in ion abundance from the ligand free protein to the complex as shown in Figure $5 \mathrm{c}$ for the peptide ion at $\mathrm{m} / \mathrm{z}$ 1735.9). The fragment ion at m/z 1909.9 (a.a. L335-K350) is first observed in the digest of the complex at $20 \mathrm{~min}$, but is not observed until $40 \mathrm{~min}$ in the digest of TetC and remains at an elevated abundance in the TetC-doxorubicin complex until $110 \mathrm{~min}$ of digestion. This indicates 

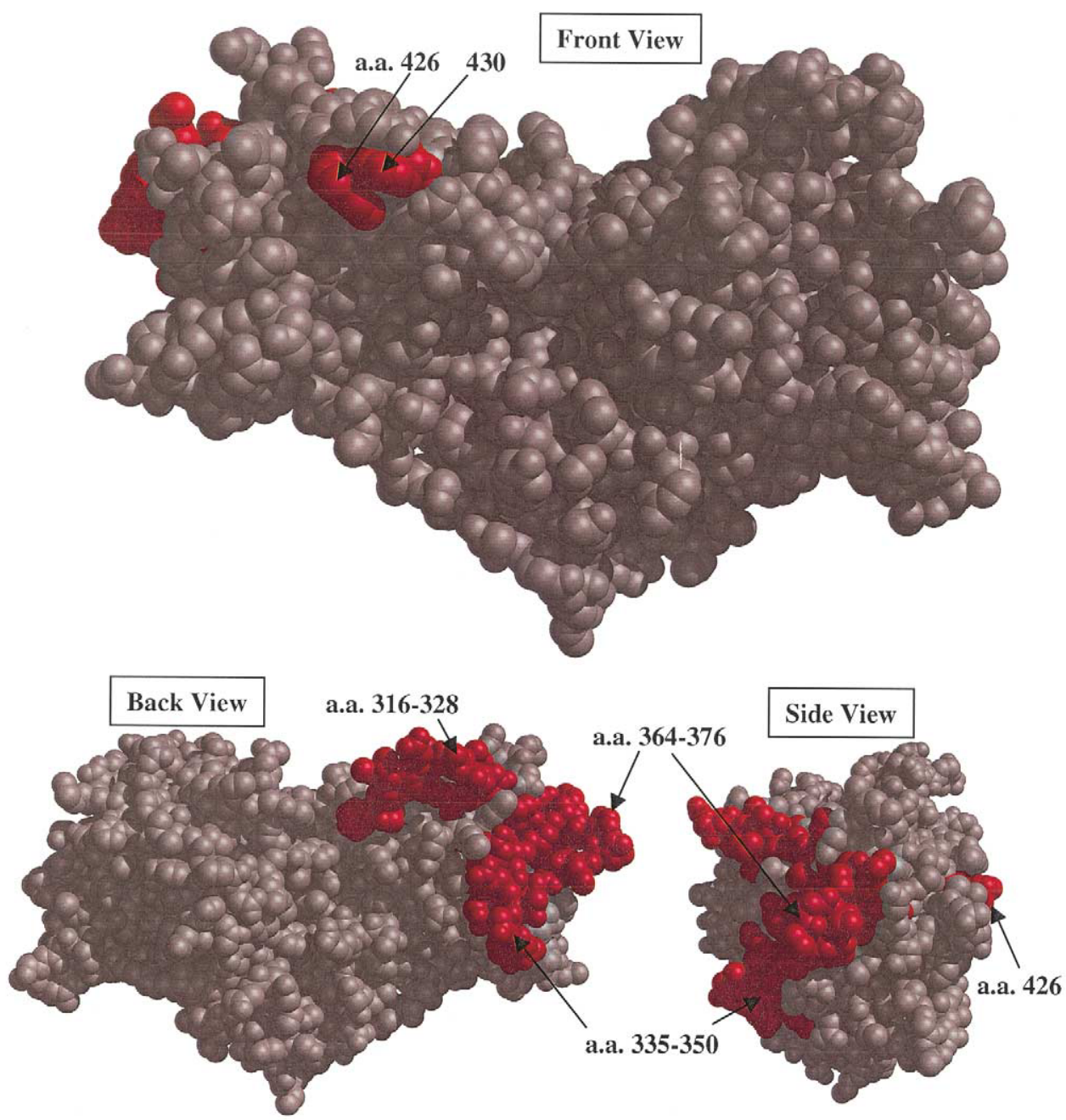

Figure 9. Space-filling molecular representation of TetC where the amino acids highlighted in red correspond to the tryptic peptides of increased abundance in the TetC-doxorubicin complex relative to TetC, including ions at $m / z 1909.9$ (a.a. 335-350), 1291.7 (a.a. 364-375), 1419.9 (a.a. 364-376), and 1582.8 (a.a. $316-328)$.

some amino acids become more accessible to trypsin upon complex formation. An interpretation of an increase in trypsin's ability to cleave at specific amino acids in the complex may suggest that doxorubicin induces a tertiary structure realignment near the binding site. The amino acids highlighted in red in Figure 9, with the exception of 426 and 430 , are the remaining five peptides showing an increased ion abundance in the complex. The peptide ions containing amino acids L335-K350 and V364-K376 are at the N- and C-terminal ends, respectively, of the peptide ion (a.a.351-R360) that had a reduced ion abundance in the TetC-doxorubicin complex. Thus, these regions of increased ion abundance border regions on TetC where a decrease in ion abundance is observed, suggesting that doxorubicin does not merely bind to the surface of TetC, but rather doxorubicin forces the protein to react, whereby the amino acids adjacent to the binding site "pucker" around doxorubicin, opening up the structure for trypsin to attack other sites more readily.

\section{Conclusions}

The time resolved limited proteolysis experiments followed by MALDI MS analysis of the resulting peptide maps demonstrated herein are the first accounts of MS providing a rapid method for characterizing binding sites and protein conformation changes in protein-small molecule non-covalent complexes with $\mu \mathrm{M}$ dissociation constants. Up to this point X-ray crystallography and NMR have been the only analytical tools used to probe protein structural motifs in small molecule binding events. Although the methods outlined in this paper for a TetC-doxorubicin complex do not obtain the atomiclevel resolution of X-ray crystallography or NMR, they do provide a high throughput tool with relatively low 
amounts of protein $(4 \mu \mathrm{M})$ for determining ligand binding sites and changes in protein structure induced by the non-covalent binding of small molecule ligands. Trypsin was the enzyme used to define the time resolved limited proteolysis experimental protocol, however, we have preliminary results using other proteases including Asp-N, Glu-C, and Lys-C that corroborate the doxorubicin binding site and conformational changes induced by binding.

There are several cases where the methods described herein could be critical to investigators designing drugs to inhibit protein function or designing molecules for molecular recognition applications. One such case is the need to determine small molecule binding sites where the binding site is experimentally unknown but the $\mathrm{X}$-ray crystal structure of the protein is known as was the case detailed in this paper. This method could also prove useful in determining whether protein conformational changes induced by one ligand binding event inhibits binding of another ligand to another site. For example, we have preliminary evidence to suggest that when doxorubicin binds to $\mathrm{Tet} C$, the conformational change of TetC reduces the ability of TetC to recognize gangliosides GD1b, the TetC receptor on the cell surface of neurons.

The strengths of the time resolved limited proteolysis lie in the ability to identify ligand binding sites one ligand at a time in a relatively high throughput and automated manner using MALDI TOF MS; however there are several weaknesses to this method. The choice of MALDI TOF MS and the inherent error of $\pm 20 \%$ in ion abundance measurements could affect experiment outcome and data interpretation. For example, there may be more proteolyitic fragments that have subtle changes in abundance that are not accounted for using MALDI. For the TetC-doxorubicin complex, a 1:10 molar ratio was required to observe a change in the rate of proteolysis using MALDI, yet it is possible that this molar ratio could be reduced if the error in ion abundance measurements could be reduced. Reducing the ion abundance measurement errors could be accomplished by implementing LC/MS analysis of the time resolved proteolytic fragments. Reducing the molar ratio closer to 1:1 could also be critical in determining binding specificity of which the methods as outlined within the manuscript do not attempt to understand. In addition, if a ligand does not directly interact with amino acids consistent with a protease's specificity, then no binding site information can be obtained.

\section{Acknowledgments}

The authors acknowledge the efforts of Bill Romine at LLNL who facilitated this research by writing data analysis software that was critical to the development of high throughput time resolved limited proteolysis experiments. This research was supported by the Laboratory Directed Research and Development Program at LLNL and performed under the auspices of the U.S. Department of Energy by University of California, Lawrence Livermore National Laboratory under contract no. W-7405-ENG-48.

\section{References}

1. Veenstra, T. D. Electrospray Ionization Mass Spectrometry in the Study of Biomolecular Non-Covalent Interactions. Biophys. Chem. 1999, 79, 63-79.

2. Pramanik, B. N.; Bartner, P. L.; Mirza, U. A.; Liu, Y. H. Ganguly A. K. Electrospray Ionization Mass Spectrometry for the Study of Non-Covalent Complexes: An Emerging Technology. J. Mass Spectrom. 1998, 33, 911-920.

3. Loo, J. A. Studying Non-Covalent Protein Complexes by Electrospray Ionization Mass Spectrometry. Mass Spectrom. Rev. 1997, 16, 1-23.

4. Wu, Q. Y.; Gao, J. M.; Joseph-McCarthy, D; Sigal, G. B.; Bruce, J. E.; Whitesides, G. M.; Smith, R. D. Carbonic AnhydraseInhibitor Binding: From Solution to the Gas Phase. J. Am. Chem. Soc. 1997, 119, 1157-1158.

5. Wigger, M.; Eyler, J. R.; Benner, S. A.; Li, W. Q.; Marshall, A. G. Fourier Transform-Ion Cyclotron Resonance Mass Spectrometric Resolution, Identification, and Screening of Non-Covalent Complexes of hck src Homology 2 Domain Receptor and Ligands from a 324-Member Peptide Combinatorial Library. J. Am. Soc. Mass Spectrom. 2002, 13, 1162-1169.

6. Cancilla, M. T.; Leavell, M. D.; Chow, J.; Leary, J. A. Mass Spectrometry and Immobilized Enzymes for the Screening of Inhibitor Libraries. Proc. Natl. Acad. Sci. U.S.A. 2000, 97, 12008-12013.

7. van Breemen, R. B.; Huang, C. R.; Nikolic, D.; Woodbury, C. P.; Zhao, Y. Z.; Venton, D. L. Pulsed Ultrafiltration Mass Spectrometry: A New Method for Screening Combinatorial Libraries. Anal. Chem. 1997, 69, 2159-2164.

8. Cai, J. Y.; Henion, J. On-Line Immunoaffinity ExtractionCoupled Column Capillary Liquid Chromatography Tandem Mass Spectrometry: Trace Analysis of LSD Analogs and Metabolites in Human Urine. Anal. Chem. 1996, 68, 72-78.

9. Nedved, M. L.; HabibiGoudarzi, S.; Ganem, B.; Henion, J. D. Characterization of Benzodiazepine "Combinatorial" Chemical Libraries by On-Line Immunoaffinity Extraction, Coupled Column HPLC Ion Spray Mass Spectrometry Tandem Mass Spectrometry. Anal. Chem. 1996, 68, 4228-4236.

10. Siegel, M. M.; Tabei, K.; Bebernitz, G. A.; Baum, E. Z. Rapid Methods for Screening Low Molecule Mass Compounds NonCovalently Bound to Proteins Using Size Exclusion and Mass Spectrometry Applied to Inhibitors of Human Cytomegalovirus Protease. J. Mass Spectrom. 1998, 33, 264-273.

11. Moy, F. J.; Haraki, K.; Mobilio, D.; Walker, G.; Powers, R.; Tabei, K.; Tong, H.; Siegel, M. M. MS/NMR: A structureBased Approach for Discovering Protein Ligands and for Drug Design by Coupling Size Exclusion Chromatography, Mass Spectrometry, and Nuclear Magnetic Resonance Spectroscopy. Anal. Chem. 2001, 73, 571-581.

12. Young, M. M.; Tang, N.; Hempel, J. C.; Oshiro, C. M.; Taylor, E. W.; Kuntz, I. D.; Gibson, B. W.; Dollinger, G. High Throughput Protein Fold Identification by Using Experimental Constraints Derived from Intramolecular Cross- Links and Mass Spectrometry. Proc. Natl. Acad. Sci. U.S.A. 2000, 97, 5802-5806.

13. Bennett, K. L.; Kussmann, M.; Bjork, P.; Godzwon, M.; Mikkelsen, M.; Sorensen, P.; Roepstorff, P. Chemical Cross Linking with Thiol-Cleavable Reagents Combined with Differential Mass Spectrometric Peptide Mapping-a Novel Approach to Assess Intermolecular Protein Contacts. Protein Sci. 2000, 9, 1503-1518.

14. Rappsilber, J.; Siniossoglou, S.; Hurt, E. C.; Mann, M. A Generic Strategy to Analyze the Spatial Organization of MultiProtein Complexes by Cross Linking and Mass Spectrometry. Anal. Chem. 2000, 72, 267-275.

15. Sinz, A.; Wang, K. Mapping Protein Interfaces with a Fluorogenic Cross Linker and Mass Spectrometry: Application to 
Nebulin-Calmodulin Complexes. Biochemistry 2001, 40, 79037913.

16. Cai, K.; Itoh, Y.; Khorana, F. C. Mapping of Contact Sites in Complex Formation Between Transducin and Light-Activated Rhodopsin by Covalent Cross Linking: Use of a Photoactivatable Reagent. Proc. Natl. Acad. Sci. U.S.A. 2001, 98, 4877-4882.

17. Shapiro, R. E.; Specht, C. D.; Collins, B. E.; Woods, A. S.; Cotter, R. J.; Schnaar, R. L. Identification of a Ganglioside Recognition Domain of Tetanus Toxin Using a Novel Ganglioside Photoaffinity Ligand. J. Biol. Chem. 1997, 272, 3038030386.

18. Seielstad, D. A.; Carlson, K. E.; Kushner, P. J.; Greene, G. L.; Katzenellenbogen, J. A. Analysis of the Structural Core of the Human Estrogen-Receptor Ligand-Binding Domain by Selective Proteolysis Mass Spectrometric Analysis. Biochemistry 1995, 34, 12605-12615.

19. Leite, J. F.; Amoscato, A. A.; Cascio, M. Coupled Proteolytic and Mass Spectrometry Studies Indicate a Novel Topology for the Glycine Receptor. J. Biol. Chem. 2000, 275, 13683-13689.

20. Kriwacki, R. W.; Wu, J.; Siuzdak, G.; Wright, P. E. Probing Protein-Protein Interactions with Mass Spectrometry and Isotopic Labeling: Analysis of the p21/cdk2 Complex. J. Am. Chem. Soc. 1996, 118, 5320-5321.

21. Zhao, Y. M.; Muir, T. W.; Kent, S. B. H.; Tischer, E.; Scardina, J. M.; Chait, B. T. Mapping Protein-Protein Interactions by Affinity-Directed Mass Spectrometry. Proc. Natl. Acad. Sci. U.S.A. 1996, 93, 4020-4024.

22. Zhao, Y. M.; Chait, B. T. Protein Epitope Mapping by Mass Spectrometry. Anal. Chem. 1994, 66, 3723-3726.

23. Gervasoni, P.; Staudenmann, W.; James, P.; Pluckthun, A. Identification of the Binding Surface on $\beta$-Lactamase for Groel by Limited Proteolysis and MALDI Mass Spectrometry. Biochemistry 1998, 37, 11660-11669.

24. Spolaore, B.; Bermejo, R.; Zambonin, M.; Fontana, A. Protein Interactions Leading to Conformational Changes Monitored by Limited Proteolysis: Apo Form and Fragments of Horse Cytochrome c. Biochemistry 2001, 40, 9460-9468.

25. Cohen, S. L.; Ferredamare, A. R.; Burley, S. K.; Chait, B. T. Probing the Solution Structure of the DNA-Binding Protein Max by a Combination of Proteolysis and Mass Spectrometry. Protein Sci. 1995, 4, 1088-1099.

26. Schechter, I.; Berger, A. On the Size of the Active Site in Proteases. Biochem. Biophys. Res. Commun. 1967, 27, 157-162.

27. Halpern, J. L.; Loftus, A. Characterization of the ReceptorBinding Domain of Tetanus Toxin. J. Biol. Chem. 1993, 268, 11188-11192.

28. Montecucco, C. Clostridial Neurotoxins. The Molecular Pathogenesis of Tetanus and Botulism, Vol. XCV. Springer-Verlag: Berlin, 1995, pp 257-274.

29. Lightstone, F. C.; Prieto, M. C.; Singh, A. K.; Piqueras, M. C.; Whittal, R. M.; Knapp, M. S.; Balhorn, R.; Roe, D. C. Identification of Novel Small Molecule Ligands that Bind to Tetanus Toxin. Chem. Res. Toxicol. 2000, 13, 356-362.

30. Cosman, M.; Lightstone, F. C.; Krishnan, V. V.; Zeller, L.; Prieto, M. C.; Roe, D. C. Balhorn R. Identification of Novel
Small Molecules that Bind to Two Different Sites on the Surface of Tetanus Toxin c Fragment. Chem. Res. Toxicol. 2002, $15,1218-1228$.

31. Dai, Y. Q.; Whittal, R. M.; Li, L. Confocal Fluorescence Microscopic Imaging for Investigating the Analyte Distribution in MALDI Matrices. Anal. Chem. 1996, 68, 2494-2500.

32. Onnerfjord, P.; Ekstrom, S.; Bergquist, J.; Nilsson, J.; Laurell, T.; Marko-Varga, G. Homogeneous Sample Preparation for Automated High Throughput Analysis with MatrixAssisted Laser Desorption/Ionization Time-of-Flight Mass Spectrometry. Rapid Commun. Mass Spectrom. 1999, 13, 315322.

33. Edmondson, R. D.; Russell, D. H. Evaluation of MatrixAssisted Laser dDesorption Ionization Time-of-Flight Mass Measurement Accuracy by Using Delayed Extraction. J. Am. Soc. Mass Spectrom. 1996, 7, 995-1001.

34. Dai, Y. Q.; Whittal, R. M.; Li, L. Two-Layer Sample Preparation: A Method for MALDI MS Analysis of Complex Peptide and Protein Mixtures. Anal. Chem. 1999, 71, 1087-1091.

35. Rong, S. B.; Zhang, J.; Neale, J. H.; Wroblewski, J. T.; Wang, S.; Kozikowski, A. P. Molecular Modeling of the Interactions of Glutamate Carboxypeptidase ii with its Potent naag-Based Inhibitors. J. Med. Chem. 2002, 45, 4140-4152.

36. Brady, G. P.; Stouten, P. F. W. Fast Prediction and Visualization of Protein Binding Pockets with Pass. J. Comput.-Aided Molec. Des. 2000, 14, 383-401.

37. Hendlich, M.; Rippmann, F.; Barnickel, G. Ligsite: Automatic and Efficient Detection of Potential Small Molecule-Binding Sites in Proteins. J. Mol. Graph. 1997, 15, 359.

38. Veenstra, T. D.; Johnson, K. L.; Tomlinson, A. J.; Naylor, S.; Kumar, R. Determination of Calcium-Binding Sites in Rat Brain Calbindin d-28k by Electrospray Ionization Mass Spectrometry. Biochemistry 1997, 36, 3535-3542.

39. Loo, J. A.; Loo, R. R. O.; Udseth, H. R.; Edmonds, C. G.; Smith, R. D. Solvent-Induced Conformational-Changes of Polypeptides Probed by Electrospray Ionization Mass Spectrometry. Rapid Commun. Mass Spectrom. 1991, 5, 101-105.

40. Mirza, U. A.; Cohen, S. L.; Chait, B. T. Heat-Induced Conformational Changes in Proteins Studied by Electrospray Ionization Mass Spectrometry. Anal. Chem. 1993, 65, 1-6.

41. Mirza, U. A.; Chait, B. T. Effects of Anions on the Positive-Ion Electrospray Ionization Mass Spectra of Peptides and Proteins. Anal. Chem. 1994, 66, 2898-2904.

42. Vis, H.; Heinemann, U.; Dobson, C. M.; Robinson, C. V. Detection of a Monomeric Intermediate Associated with Dimerization of Protein hu by Mass Spectrometry. J. Am. Chem. Soc. 1998, 120, 6427-6428.

43. Rogniaux, H.; van Dorsselaer, A.; Barth, P.; Biellmann, J. F.; Barbanton, J.; van Zandt, M.; Chevrier, B.; Howard, E.; Mitschler, A.; Potier, N.; Urzhumtseva, L.; Moras, D.; Podjarny, A. Binding of Aldose Reductase Inhibitors: Correlation of Crystallographic and Mass Spectrometric Studies. J. Am. Soc. Mass Spectrom. 1999, 10, 635-647. 